\title{
Manipulation of growth modes in heteroepitaxy: $\mathrm{Ni} / \mathrm{Cu}(\mathbf{1 1 1})$
}

\author{
Wulf Wulfhekel, Ingo Beckmann, Nuphar N. Lipkin, ${ }^{\text {a) }}$ Georg Rosenfeld, Bene \\ Poelsema, ${ }^{\text {b) }}$ and George Comsa \\ Institut für Grenzflächenforschung und Vakuumphysik, Forschungszentrum Jülich GmbH, 52425 Jülich, \\ Germany
}

(Received 27 August 1996; accepted for publication 7 October 1996)

\begin{abstract}
Growth manipulation methods, which have been successfully used to improve the growth of homoepitaxial films, are applied to molecular beam epitaxy of the heteroepitaxial system $\mathrm{Ni} / \mathrm{Cu}(111)$. The procedures applied are temperature reduction during nucleation and pulsed ion bombardment during deposition. While the first does not lead to smoother films, the ion beam assisted growth is successful in reducing the film roughness. (C) 1996 American Institute of
\end{abstract} Physics. [S0003-6951(96)03949-6]

In this letter we report on experiments performed to test whether recently developed methods to improve the growth of homoepitaxial systems ${ }^{1,2}$ can be successfully applied to heteroepitaxial systems. These methods are based on the idea that layer-by-layer growth can be induced by creating an enhanced density of nuclei during the early stage of growth of each monolayer. In this way the nucleation of islands in the second layer is suppressed until coalescence of the islands of the first layer takes place: the island density is increased and thus the island dimensions before coalescence are reduced so that atoms deposited on top of these islands visit the island edge at an enhanced rate leading to an improved interlayer mass transport. Clearly, this recipe only works if the island density is enhanced in a way that does not reduce the atom mobility on top of islands. The general growth recipe can be realized by different techniques, two of which will be used in this letter. An enhanced island density can be produced without affecting the atom mobility on top of the islands during island growth by lowering the substrate temperature during the nucleation stage of each monolayer but continuing deposition at a higher temperature (temperature alternation technique). The same effect can be achieved by gently bombarding the growing surface with low energy ions during nucleation but shutting off the ion beam while the rest of the monolayer is deposited (pulsed ion beam assisted deposition). These procedures have been successfully applied to the homoepitaxial systems $\operatorname{Ag} / \operatorname{Ag}(111),{ }^{3}$ $\mathrm{Cu} / \mathrm{Cu}(111)^{4}$ and $\mathrm{Pt} / \mathrm{Pt}(111) .^{5}$ It is the aim of the work described in this letter to check whether these procedures which ultimately rely on the control of growth kinetics are also suited for heteroepitaxial systems.

As a heteroepitaxial test system we have chosen Ni/ $\mathrm{Cu}(111)$. It was reported that due to the small misfit of only $2.5 \%$, Ni grows pseudomorphically up to coverages of 7 monolayers (ML). ${ }^{6-8}$ No stacking faults at the interface could be observed. ${ }^{8,9}$ Huang and coworkers reported ${ }^{10}$ a lack of reflection high-energy electron diffraction (RHEED) oscillations and a decay of the RHEED intensity during growth indicating that growth does not proceed in a layer-by-layer manner and the films roughen with thickness. Early experi-

\footnotetext{
a) Present address: RAFAEL (Dep. 27), P.O. Box 2250, Haifa 31021, Israel. ${ }^{b)}$ Faculty of Applied Physics and Centre for Materials Research (CMO), University of Twente, P.O. Box 217, 7500 AE Enschede, the Netherlands.
}

ments of Gradmann ${ }^{6}$ showed, that flat films can be grown at elevated temperatures (around $470 \mathrm{~K}$ ). However, from electron energy loss spectroscopy of $\mathrm{CO}$ adsorbed on $\mathrm{Ni} / \mathrm{Cu}(111)$ it was concluded that already during the early stages of growth, intermixing of $\mathrm{Ni}$ and $\mathrm{Cu}$ starts at the interface at temperatures as low as $375 \mathrm{~K} .{ }^{11}$ To avoid this intermixing, all experiments described here were performed below this temperature.

The experiments were carried out in an ultra-highvacuum chamber with base pressure below $3 \times 10^{-11}$ mbar. A $67 \mathrm{meV}$ supersonic He beam was used for He scattering (TEAS) measurements. The scattering apparatus was also equipped with an Auger electron spectrometer (AES) and has been described in detail elsewhere. ${ }^{12,13}$

The $\mathrm{Cu}(111)$ sample was prepared by repeated cycles of sputtering and annealing until no contamination could be detected by means of AES and the mean terrace width on the surface was over $1000 \AA^{4}{ }^{4} \mathrm{Ni}$ was evaporated onto the sample from a high purity, thoroughly outgassed Ni-disk heated by electron bombardment. During deposition the pressure stayed below $1 \times 10^{-10}$ mbar.

The growth of the Ni film was monitored by recording the specularly reflected $\mathrm{He}$ intensity during deposition. The scattering condition was set to anti-phase $(n=2.5)$ with respect to the $\mathrm{Ni} / \mathrm{Cu}$ step height as detected by TEAS. ${ }^{14}$ Under this condition the specularly reflected intensity is determined by the height distribution of the deposited material. During layer-by-layer growth (2D-growth), the intensity shows oscillations with a period corresponding to the deposition of one monolayer while for three-dimensional (multilayer) growth, a monotonically decaying intensity is observed. ${ }^{12}$

Figure 1 shows the normalized $\mathrm{He}$ intensities recorded for different substrate temperatures during deposition of $\mathrm{Ni}$ at a rate of $R=0.02 \mathrm{ML} / \mathrm{s}$. At low temperatures the intensity drops monotonically indicative of multilayer growth. For growth temperatures above $250 \mathrm{~K}$, a single, weak oscillation is observed followed again by a monotonic decay. The first layer obviously grows in an imperfect two-dimensional manner. However, successive layers grow three-dimensionally as indicated by the decay. With rising temperature, the oscillation becomes more pronounced and the following decay becomes slower indicating that growth is smoother at higher temperatures as expected. However, at none of the temperatures layer-by-layer growth was observed. 


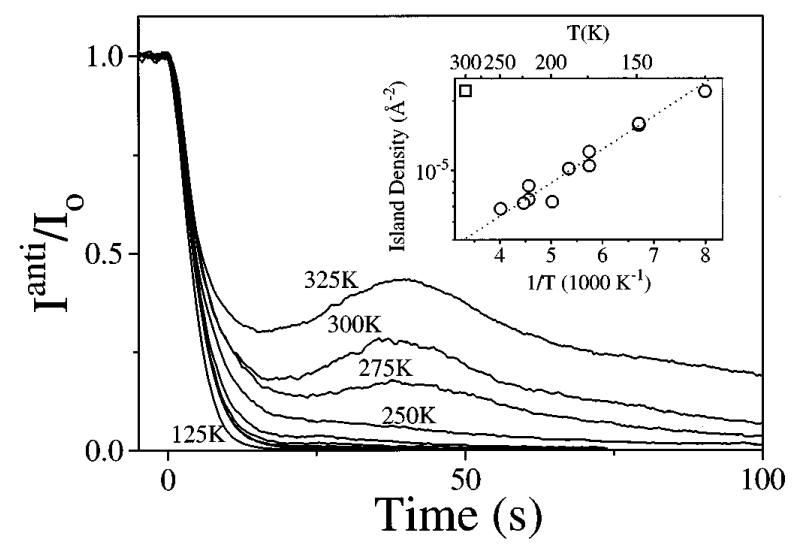

FIG. 1. Evolution of the normalized specular He intensity under anti-phase conditions $(n=2.5)$ during deposition of $\mathrm{Ni}$ onto $\mathrm{Cu}(111)$ at substrate temperatures between 125 and $325 \mathrm{~K}$ (in steps of $25 \mathrm{~K}$ ). Deposition was carried out at a rate of $R=0.02 \mathrm{ML} / \mathrm{s}$. The inset displays an Arrhenius plot of the mean island density as a function of temperature, obtained from the broadening of anti-phase peak profiles as a result of conventional nucleation (circles) and nucleation after ion bombardment (square).

Also the island density as a function of temperature was estimated and found to behave as expected. In the experiments $0.3 \mathrm{ML} \mathrm{Ni}$ were deposited with a rate of $0.02 \mathrm{ML} / \mathrm{s}$ at temperatures between 135 and $260 \mathrm{~K}$ followed by cooling of the crystal to $\approx 120 \mathrm{~K}$ to suppress further diffusion. The resulting island densities were estimated from a fit to the broadened anti-phase peak profiles taken at that temperature, according to the method described in Ref. 15. The inset of Fig. 1 displays an Arrhenius plot of the island number density. With rising temperature a decrease in the island number density is observed, as expected from classical nucleation theory. ${ }^{16}$

On the basis of this result, an attempt was made to influence the growth of $\mathrm{Ni}$ on $\mathrm{Cu}(111)$ by variation of the substrate temperature during growth. In a first step just enough material was brought onto the surface to accomplish the stage of nucleation $(0.1 \mathrm{ML})$ at low temperatures ranging from 70 to $220 \mathrm{~K}$. The deposition was continued at higher temperatures between 200 and $300 \mathrm{~K}$. Provided that the nuclei survive the heating up to the second temperature, the artificially raised island number density on the surface should result in an improved interlayer mass transport. However, none of the various deposition curves displayed clear oscillations as have been seen with the same procedure for $\mathrm{Ag} / \mathrm{Ag}(111)^{3}$ or $\mathrm{Cu} / \mathrm{Cu}(111) .{ }^{4}$ On the contrary, as illustrated in Fig. 2, the normalized anti-phase He intensity reflected from the surface after the second stage of growth may be even lower than during unmanipulated growth, indicating even rougher films. This discrepancy cannot be due to some anomaly in nucleation, because, as shown above, the nucleation of $\mathrm{Ni}$ on $\mathrm{Cu}(111)$ behaves as normal as that of $\mathrm{Cu}$ on $\mathrm{Cu}(111)$ or $\mathrm{Ag}$ on $\mathrm{Ag}(111)$. The reason for the discrepancy is found in an unusual annealing behavior of the $\mathrm{Ni}$ islands nucleated at low temperatures. During heating of $0.3 \mathrm{ML} \mathrm{Ni}$ on $\mathrm{Cu}(111)$, deposited at $125 \mathrm{~K}$, we recorded the reflected in-phase intensity, which relates to the defect density. As can be seen in the inset of Fig. 2, the intensity decays at 200 and $320 \mathrm{~K}$ due to some additional diffuse scattering from defects on the surface evolving during the heating. This behavior is

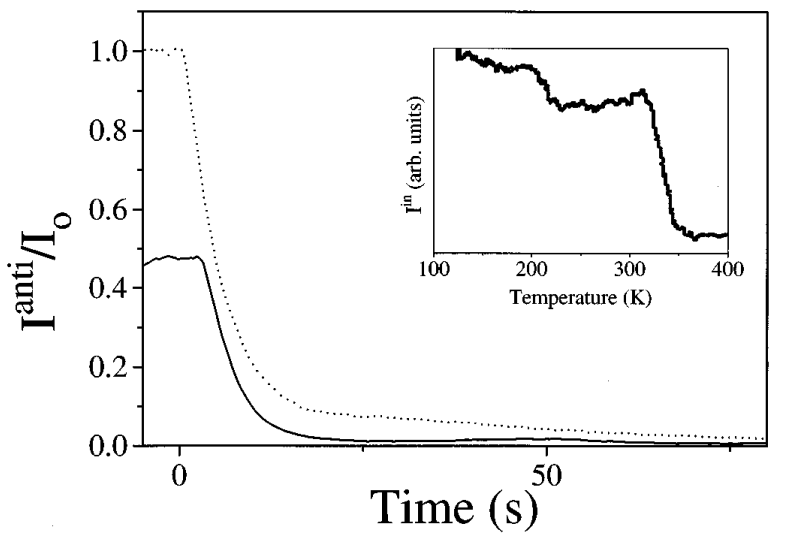

FIG. 2. Evolution of the normalized anti-phase He intensities during deposition of $\mathrm{Ni}$ at $T=245 \mathrm{~K}$ onto $\mathrm{Cu}(111)$ surfaces, which were treated by pre-deposition of $0.1 \mathrm{ML} \mathrm{Ni}$ at $135 \mathrm{~K}$ (solid line) and for comparison during unmanipulated growth at $T=250 \mathrm{~K}$ (dotted line). Deposition was carried out at a rate of $R=0.02 \mathrm{ML} / \mathrm{s}$. The inset displays the in-phase $n=2$ intensity during annealing of $0.3 \mathrm{ML} \mathrm{Ni}$, deposited onto $\mathrm{Cu}(111)$ at $125 \mathrm{~K}$, as a function of substrate temperature.

the complete opposite to the case of $\mathrm{Cu} / \mathrm{Cu}(111)$ and $\operatorname{Ag} / \operatorname{Ag}(111) .^{4,3}$ Indeed, instead of increasing, which would reflect the expected annealing, it decreases indicating a roughening of the surface, which prevents smooth growth in the second stage. To circumvent these problems we performed experiments where we chose the growth temperature below the onset of the first roughening at $200 \mathrm{~K}$ and the nucleation temperature as low as possible (70 K). However, no major improvement of growth could be observed. This is most likely due to the rather small enhancement of the island density and the low probability of $\mathrm{Ni}$ atoms to thermally overcome the step edge barrier at the island edges.

For pulsed ion beam assisted growth, freshly prepared surfaces were bombarded with a fluence $Q=4 \times 10^{17}$ ions $/ \mathrm{m}^{2}$ of $1.2 \mathrm{keV} \mathrm{Ne}{ }^{+}$-ions immediately before $\mathrm{Ni}$ deposition at a rate of $R=0.02 \mathrm{ML} / \mathrm{s}$. This ion fluence leads to an enhanced island density during deposition of $\mathrm{Ni}$ on $\mathrm{Cu}(111)$ (see inset of Fig. 1). The normalized anti-phase He intensities recorded during manipulated and unmanipulated growth at 250 and $300 \mathrm{~K}$ are shown in Figs. 3(a)-3(c). It can clearly be seen that ion bombardment in the case of growth at $250 \mathrm{~K}$ leads to one clear oscillation, i.e., a two-dimensional character of growth [Fig. 3(a)]. At $300 \mathrm{~K}$ growth manipulation strongly enhances the amplitude of the oscillation [Fig. 3(b)]. In both cases a higher reflectivity around $1 \mathrm{ML}$ coverage is obtained compared to the unmanipulated growth, indicating a higher filling of the first layer and less material in the higher layers. Therefore, the pulsed ion beam assisted deposition does produce much smoother films than conventional molecular beam epitaxy. However, in the light of possible applications of the pulsed ion beam assisted deposition, it should be discussed whether the smoother surface has to be traded for a slightly roughened film-substrate interface. The nuclei created by sputtering consist of $\mathrm{Cu}$ and not of $\mathrm{Ni}$ and also some vacancies in the substrate $\mathrm{Cu}$ layer are created. Growth on this surface might cause a rougher interface between the substrate and the film. This roughness, however, is rather small since the dose used corresponds to sputtering of only $0.08 \mathrm{ML}^{4}$ and is presumably lower than the intermixing 


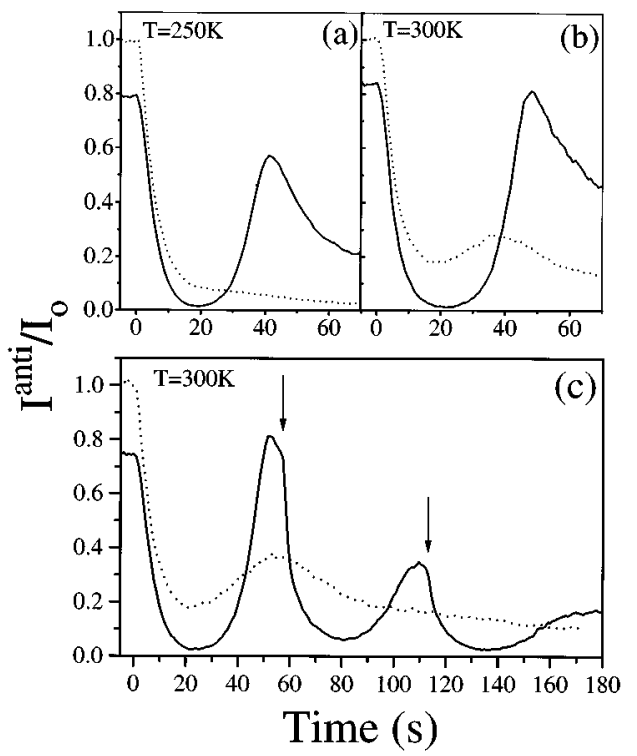

FIG. 3. Normalized anti-phase He intensities during deposition of $\mathrm{Ni}$ at temperatures as indicated onto surfaces which were treated by ion pulses with a fluence of $Q=4 \times 10^{17}$ ions $/ \mathrm{m}^{2}$ (solid lines) and for comparison during unmanipulated growth (dotted lines). Deposition was carried out at a rate of $R=0.02 \mathrm{ML} / \mathrm{s}$. (a), (b): One monolayer of Ni was deposited. (c): During deposition ion pulses were given around each monolayer completion (indicated by arrows) to subsequently grow $3 \mathrm{ML}$ of $\mathrm{Ni}$.

in the first layer observed at higher temperatures, e.g., at $375 \mathrm{~K},{ }^{11}$ needed for a smooth film surface in unmanipulated growth.

An advantage of growth manipulation by ion bombardment is the easy applicability to the growth of several monolayers. Figure 3(c) displays the anti-phase He-intensity during deposition of $3 \mathrm{ML} \mathrm{Ni}$, where an ion pulse was given during deposition just when growth in a given layer started, as indicated by the arrows. In this way the island density is enhanced in each layer and the film grows two-dimensionally. It can clearly be seen that the anti-phase intensity upon monolayer completion for manipulated growth is higher for all three layers. The unmanipulated film grows in a three dimensional mode which leads to many open layers even in thin films, whereas the manipulated film shows oscillations of the reflected $\mathrm{He}$ intensity indicative of nucleation, growth and coalescence of islands, i.e., two dimensional growth. This results in less open layers and a much higher reflected anti-phase intensity.

In conclusion it was found that growth manipulation methods, which are based on a kinetic growth model and work well for homoepitaxy, can successfully be used in heteroepitaxy although additional effects may limit the applicability of some techniques to enhance the island number density.

N. N. Lipkin acknowledges support by the Alexander von Humboldt Foundation.

${ }^{1}$ G. Rosenfeld, B. Poelsema, and G. Comsa, J. Cryst. Growth 151, 230 (1995).

${ }^{2}$ G. Rosenfeld, N. N. Lipkin, W. Wulfhekel, J. Kliewer, K. Morgenstern, B. Poelsema, and G. Comsa, Appl. Phys. A 61, 455 (1995).

${ }^{3}$ G. Rosenfeld, R. Servaty, C. Teichert, B. Poelsema, and G. Comsa, Phys. Rev. Lett. 71, 895 (1993).

${ }^{4}$ W. Wulfhekel, N. Lipkin, J. Kliewer, G. Rosenfeld, L. Jorritsma, B. Poelsema, and G. Comsa, Surf. Sci. 348, 227 (1996).

${ }^{5}$ S. Esch, M. Breeman, M. Morgenstern, T. Michely, and G. Comsa, Surf. Sci. 365, 187 (1996).

${ }^{6}$ U. Gradmann, Ann. Phys. (Leipzig) 13, 213 (1964).

${ }^{7}$ D. W. Gidley, Phys. Rev. Lett. 62, 811 (1989).

${ }^{8}$ J. Zhang, Z. L. Han, and S. Varma, Surf. Sci. 298, 351 (1993).

${ }^{9}$ S. P. Tear and K. Röll, J. Phys. C 15, 5521 (1982).

${ }^{10}$ F. Huang, M. T. Kief, G. J. Mankey, and R. F. Willis, Phys. Rev. B 49, 3962 (1994).

${ }^{11}$ S. Yang, M. Yu, G. Meigs, X. H. Feng, and E. Garfunkel, Surf. Sci. 205, L777 (1988).

${ }^{12}$ B. Poelsema and G. Comsa, Scattering of Thermal Energy Atoms, Springer Tracts in Modern Physics (Springer, Berlin, 1989), Vol. 115

${ }^{13}$ B. Poelsema, G. Mechtersheimer, and G. Comsa, Surf. Sci. 111, 519 (1981).

${ }^{14}$ The scattering angle for anti-phase conditions was determined from the minima in the angular dependence of the specularly reflected He intensity of $0.3 \mathrm{ML}$ of $\mathrm{Ni}$ on $\mathrm{Cu}(111)$.

${ }^{15}$ M. Henzler, Appl. Surf. Sci. 11/12, 450 (1982); J. Wollschläger, J. Falta, and M. Henzler, Appl. Phys. A 50, 57 (1990).

${ }^{16}$ J. A. Venables, Philos. Mag. 27, 697 (1973). 